REPORT

\title{
DREAMing about arthritic pain
}

\author{
H-Y M Cheng, J M Penninger
}

Ann Rheum Dis 2004;63(Suppl II):ii72-ii75. doi: 10.1136/ard.2004.029942

The experience of acute pain serves a crucial biological purpose: it alerts a living organism to environmental dangers, inducing behavioural responses which protect the organism from further damage. In contrast, chronic pain arising from disease states and/or pathological functioning of the nervous system offers no advantage and may be debilitating to those afflicted. Despite recent advances in our understanding of pain mechanisms, the satisfactory management of pathological pain eludes current treatment strategies. We have demonstrated in a previous study on dream deficient mice the pivotal role of downstream regulatory element antagonistic modulator (DREAM) in modulating pain sensitivity in a number of behavioural models, including acute and chronic neuropathic pain. DREAM is a novel calcium binding transcriptional repressor for the prodynorphin gene in spinal cord neurones. The marked attenuation in pain behaviour exhibited by dream ${ }^{-/-}$mice was shown, by pharmacological and biochemical analyses, to be due to increased activation of the endogenous $\kappa$-opioid system. Importantly, loss of DREAM also attenuated inflammatory pain. Thus, DREAM and the DREAM pathway constitute a novel therapeutic paradigm for the treatment of chronic pain in arthritis.

$\mathrm{T}$ he ability to feel pain is essential for the survival and wellbeing of organisms and alerts the organism to imminent danger. The response to pain allows us to prevent or minimise the injury. Discrete pathways within the somatosensory system are dedicated to the many aspects of processing of noxious information. Clinical pain arises when the somatosensory system is altered so that the nature of the sensory input is no longer faithfully represented. The symptoms can be:

- spontaneous pain in the absence of any sensory input

- heightened or exaggerated pain in response to a normally noxious stimulus (hyperalgesia)

- pain in response to triggers which do not normally provoke pain (allodynia) and

- symptoms of sensory deficits.

Chronic pain such as neuropathic pain in nerve injuries poses a major challenge to clinicians because in different patients multiple and diverse mechanisms come into play.

Arthritis in humans and in animal models is characterised by synovial inflammation and erosion of bone and cartilage and is ultimately a lifelong crippling disease. ${ }^{1}$ Clinically, one of the most important and destructive manifestations in patients with arthritis is chronic and severe joint pain. In this report we will discuss new advances in pain research that might allow better understanding and treatment of chronic pain.

\section{PAIN MECHANISMS IN RHEUMATOID ARTHRITIS}

Rheumatoid arthritis (RA) is a progressively degenerative disease characterised by recurrent inflammation and eventual destruction of the synovial tissue, cartilage, and juxtaarticular bone of a joint. ${ }^{1}$ The synovium and joint capsule are densely innervated not only by postganglionic sympathetic nerve fibres but also by peripheral afferents of dorsal root ganglia sensory neurones, which convey sensory and nociceptive (pain) information to the central nervous system. Pain associated with RA can occur spontaneously or can be evoked by gentle stimulation of the joint when it is moved within its normal working range.

Although there is no consensus on a single causative trigger for RA, what ensues initially is a local inflammatory response mediated by the adaptive and innate immune systems, as well as resident non-immune cells-for example, synovial fibroblasts. Lymphocytes and macrophages release proinflammatory cytokines such as tumour necrosis factor and multiple interleukins including IL- 1 and IL-6. Other components of the local inflammatory reaction released by the microenvironment of the inflamed and injured tissue include histamine, bradykinin, serotonin, prostaglandin $\mathrm{E}_{2}$ $\left(\mathrm{PGE}_{2}\right)$, ATP and protons $\left(\mathrm{H}^{+}\right)$. The peripheral terminals of $\mathrm{A} \delta$ and $\mathrm{C}$ fibres, which are activated only by noxious (painful) stimuli under non-inflamed conditions, express many receptors and ion channels that recognise the various inflammatory mediators in the vicinity. Thus, the local inflammation results in the release of multiple factors that activate local nerve terminals involved in pain perception.

Some of these chemical messengers can directly activate nociceptive peripheral afferent neurones leading to propagation of action potentials to the spinal cord, while others lower the threshold of activation of these neurones (hypersensitisation). ${ }^{2}$ For instance, $\mathrm{PGE}_{2}$ can increase the excitability and decrease the threshold of nociceptor terminals by inducing the phosphorylation of tetrodotoxin resistant sodium channels. ${ }^{3}$ Non-steroidal anti-inflammatory drugs, which are commonly used to combat inflammatory disorders such as RA, act by reducing prostanoid levels. Local acidosis $\left(\mathrm{H}^{+}\right.$ release) resulting from tissue destruction can also lead to nociceptor sensitisation and/or activation via the proton sensing vanilloid receptor 1 (VRl). ${ }^{4}$ VRI can be activated by metabolites of arachidonic acid, the production of which can be induced by bradykinin acting on B2 bradykinin receptors. ${ }^{5}$ In addition, during chronic inflammation, $A \beta$ fibres, which normally respond only to tactile rather than noxious stimuli, can mediate a longlasting tactile allodynic state-that is, pain in response to innocuous touch. The heightened state of sensory/noxious processing mechanisms at the site of inflammation is referred to as peripheral sensitisation.

\footnotetext{
Abbreviations: AMPA, $\alpha$-amino-3-hydroxy-5-methyl-4isoxazolepropionic acid; CGRP, calcitonin gene related peptide; EAA, excitatory amino acid; DRE, downstream regulatory element; DREAM, downstream regulatory element antagonistic modulator; HPA, hypothalamic-pituitary-adrenal; NMDA, N-methyl-D-aspartate; $\mathrm{PGE}_{2}$, prostaglandin $\mathrm{E}_{2} ; \mathrm{RA}$, rheumatoid arthritis
} 
During inflammation, the peripheral nerve endings of nociceptive fibres release various neuromediators, namely, substance $\mathrm{P}$, calcitonin gene related peptide (CGRP), or somatostatin, into the microenvironment. These in turn can modulate the inflammatory process (neurogenic inflammation) as well as autoactivate the sensory neurones via cognate receptors expressed on the nerve terminals. For instance, substance $\mathrm{P}$ can stimulate extravasation of plasma and further recruitment of immune cells, $\mathrm{T}$ cell proliferation, mast cell degranulation, release of $\mathrm{PGE}_{2}$ and cytokines from macrophages, and proliferation of fibroblasts and endothelial cells. ${ }^{6}$ Expression of neurokinin 1 receptors which bind substance $\mathrm{P}$ is transiently upregulated in dorsal root ganglia neurones during inflammation and can promote the effects of continuous and increased local substance $\mathrm{P}$ release. ${ }^{7}$ On the other hand, other secreted neuromodulators, such as somatostatin, may act in an inhibitory feedback manner to suppress the inflammatory process and sensitisation of sensory neurones-for example, by attenuating the release of substance P. ${ }^{89}$

The autonomic nervous system is also critically involved in the pathogenesis of RA and the associated pain state. During inflammation, the hypothalamic-pituitary-adrenal (HPA) axis is activated as a stress response. ${ }^{10}$ In general, hormones released by the HPA axis (for example, cortisol, corticotrophin releasing hormone, adrenocorticotropic hormone, adrenaline (epinephrine)) have direct anti-inflammatory effects. ${ }^{10}$ Increased sympathetic tone driven by the HPA axis leads to elevated systemic and local release of the neurotransmitters noradrenaline (norepinephrine) and adenosine from sympathetic nerve terminals. Under conditions of high ligand concentration, activation by noradrenaline and adenosine of $\beta$ adrenoreceptors and A2 adenosine receptors, respectively, inhibits inflammatory processes in the synovium, whereas ligation of $\alpha 2$ adrenoreceptors and Al receptors produces the opposite, proinflammatory, response. ${ }^{11}{ }^{12}$ Sympathetic nerves can co-release endogenous opioids from their terminals and sympathetic activation can stimulate opioid release from immune cells of the inflamed tissue. ${ }^{13}{ }^{14}$ Opioid peptides $(\beta$ endorphin, met-enkephalin, dynorphin) acting on peripheral $\mu, \delta$, and/or $\kappa$-opioid receptors are antinociceptive and can prevent sensitisation of primary afferent neurones.

In RA, the functions of the HPA axis and the sympathetic nervous system are compromised over time. The low level of production of hormones by the HPA axis, rather than exerting an anti-inflammatory response, can promote and exacerbate the inflammatory process. Sympathetic nerve fibres decrease in the synovial tissue, whereas the sprouting of primary sensory afferent fibres leads to their increase in the synovium. ${ }^{15}{ }^{16}$ Nerve growth factor, a neurotrophic factor whose concentration is elevated in inflamed tissue, may play a role in the invasion of peptidergic (substance P and CGRP containing) sensory fibres in the synovial tissue, as well as increase the excitability of peripheral nociceptor terminals. ${ }^{16-18}$ Hence, perturbations of the HPA axis and sympathetic nervous system can lead to loss of or a reduction in the body's feedback inhibitory response to the peripheral inflammation, ultimately contributing to the chronic inflammatory nature of RA and promoting a protracted state of heightened pain sensitivity.

Central mechanisms also contribute to the chronic inflammatory pain state. The central terminals of peripheral sensory afferents transmit "noxious" information to secondary nociceptive neurones of the spinal dorsal horn, via the synaptic release of excitatory amino acids (EAAs), primarily glutamate and substance P. ${ }^{2}$ Receptors and channels that are activated by EAAs (for example, $N$-methyl-D-aspartate (NMDA) and $\alpha$-amino-3-hydroxy-5-methyl-4-isoxazolepropionic acid (AMPA) receptors) and substance $\mathrm{P}$ are expressed postsynaptically on dorsal horn neurones. ${ }^{2}$ Other neuromodulators, such as CGRP, neuropeptide Y, somatostatin, and $\gamma$-aminobutyric acid (GABA), may also participate in nociceptive processing at the spinal level. Normally, in a noninflamed state, central glutamate release from low threshold $\mathrm{A} \beta$ afferent nerve fibres stimulated by innocuous tactile stimuli activates AMPA receptors only. In the setting of inflammatory states such as RA, as a result of peripheral sensitisation, the same stimulus-for example, gentle pressure to the joints-is sufficient to drive massive release of glutamate and substance P from multiple afferent types (A $\beta$, $\mathrm{A} \delta, \mathrm{C})$ onto the dorsal horn: cumulative depolarisation leads to glutamate mediated NMDA receptor activation in addition to AMPA receptor activation. ${ }^{19}$ NMDA receptor activation is essential for the development of central sensitisation, which involves enhanced synaptic transmission in the spinal cord (and/or the brain) in response to noxious as well as innocuous inputs, whether or not they originate from the site of tissue injury. ${ }^{2}$

\section{THE ROLE OF DREAM IN PAIN GATING}

Downstream regulatory element antagonistic modulator (DREAM) was originally identified as a transcriptional repressor for the human prodynorphin (PDYN) gene. ${ }^{20}{ }^{21}$ An intragenic sequence termed the downstream regulatory element (DRE) within the human PDYN gene was shown to be important for controlling its basal expression as well as protein kinase A induced transactivation. ${ }^{20}$ Subsequently, using a molecular screening approach, the authors of this study demonstrated that a 284 amino acid protein with high homology to the recoverin/neuronal calcium sensor (NCS) subfamily of calcium binding proteins could bind specifically to DRE containing dsDNA oligonucleotides in vitro. ${ }^{21}$ The DREAM protein, like other members of the NCS superfamily, is comprised of four calcium binding domains known as EF hand motifs, as well as a unique $\mathrm{N}$-terminal extended sequence. Association of calcium with the EF hand domains results in a conformational change of the DREAM protein, such that its affinity for DRE DNA sequences is drastically reduced. ${ }^{22}$ Therefore, under conditions of low calcium concentration, calcium free (apo)DREAM binds tightly to the prodynorphin DRE sequence and represses PDYN gene transcription, whereas increasing calcium levels abrogate the DREAM-DRE association and permit transcription to occur.

In addition to its role in transcriptional regulation, DREAM has been implicated in other biological processes. The presenilin (PS) genes, PS1 and PS2, are frequently mutated in patients with the familial form of Alzheimer's disease. Mutations in PS1 and PS2 are believed to contribute to the formation of amyloid plaques, the biochemical hallmark of Alzheimer's disease, and may induce neurodegeneration by affecting programmed cell death or apoptosis. These events may, in turn, depend on the association of presenilins with other protein factors. DREAM, also known as calsenilin, was discovered as a binding partner for PS2. ${ }^{23}$ Overexpression of calsenilin altered the proteolytic processing of PS2 holoprotein in vitro. ${ }^{23}$ It was proposed that calsenilin may serve as the link between aberrant $\mathrm{Ca}^{2+}$ signalling and the pathogenesis of Alzheimer's disease.

Another study set out to identify binding partners for Kv4 voltage gated potassium channels. ${ }^{24}$ The transient A-type $\mathrm{K}^{+}$ current is essential for controlling the excitability of neurones and cardiac cells. The cloned Kv4 genes, when expressed in heterologous systems, recapitulate many properties of native A-type currents, but are unable to account for all features. Therefore, it was believed that auxiliary factors are required to confer specific qualities to Kv4 channel function. The authors of this study identified $\mathrm{Kv}$ channel-interacting protein 1 (KChIP1) and KChIP2 in a screen for Kv4 binding 
factors. ${ }^{24}$ DREAM, known as KChIP3, was identified by its structural homology to the other KChIPs. It was proposed that KChIPs are integral regulatory components of Kv4 channels and augment A-type currents.

Given the possible pleiotropic functions of DREAM, our laboratory disrupted the dream gene by mouse knock-out technology to investigate its in vivo function. Mice lacking the dream gene $\left(\right.$ dream $\left.^{-/}\right)$underwent normal development and exhibited no obvious anatomical defects compared with wild-type mice harbouring the intact dream gene. ${ }^{25}$ dream $^{-/-}$ mice did not display overt or profound behavioural deficiencies-for example, in tests of motor skill and coordination. Moreover, heart functions appeared normal suggesting that loss of DREAM had no detectable effect on Kv4 channels in cardiomyocytes. Thus, although it cannot be excluded, we did not observe any evidence for an important function of DREAM in behavioural studies or the function of $\mathrm{K}^{+}$ channels.

One of the consistent and distinguishing features of dream $^{-/}$mice is their hypoalgesic response in all pain paradigms employed ${ }^{25}$ (fig 1). Sensory perception to innocuous touch stimuli was unaffected in dream $^{-/}$mice under basal conditions, but pain behaviour elicited by noxious heat or mechanical pressure was greatly reduced. dream ${ }^{-/}$mice also displayed attenuated pain behaviour in two models of visceral pain (intraperitoneal $\mathrm{MgSO}_{4}$ and acetic acid). Subcutaneous formalin injection into the hind paw, a model of tonic pain involving tissue injury and inflammation, elicited less pain behaviour in dream $^{-/-}$mice, compared with wild-type controls, in both the early and late phases following injection. Pain behaviour associated with the early phase of the formalin test is often attributed to direct activation of peripheral afferents, whereas central sensitisation in combination with ongoing afferent activity plays a more prominent role in the late phase.

We also assessed pain evoked by subcutaneous injection of capsaicin and carrageenan, models of neurogenic and nonneurogenic inflammation, respectively. ${ }^{25}$ When injected into the hind paw, both agents produced a diminished state of

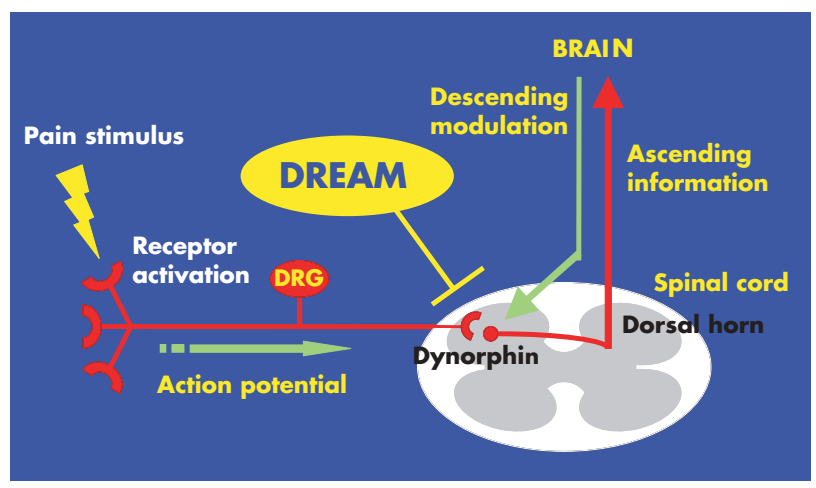

Figure 1 A model for downstream regulatory element antagonistic modulator (DREAM) dependent modulation of pain transmission at the spinal level. Noxious stimulation of peripheral tissues activates peripheral sensory afferents and evokes the release of excitatory neurotransmitters from their central terminals onto the spinal dorsal horn. The nociceptive information is eventually relayed to higher pain processing centres within the brain, which, in turn, can provide descending modulation of nociceptive transmission at the spinal level. Generally speaking, spinal opioids such as dynorphin have inhibitory effects on nociceptive transmission. DREAM is expressed in the spinal cord and controls the expression of the prodynorphin gene. Loss of DREAM function-for example, by genetic deletion in our mouse model-results in elevated expression of prodynorphin and enhanced $\kappa$-opioid receptor activation under basal conditions. As a consequence, DREAM deficient mice exhibit a phenotype of "ongoing analgesia". DRG, dorsal root ganglion. mechanical hypersensitivity (allodynia) in $\mathrm{dream}^{-/}$mice compared with wild-type controls. As in the acute phase of the formalin test, the acute pain response following capsaicin injection was reduced in dream $^{-/}$mice. In the carrageenan test, it was noted that only the magnitude of the allodynia, and not the kinetics of recovery from the allodynic state, was altered in the absence of DREAM. By using paw thickness as an indication of the inflammatory process, we found $\mathrm{dream}^{-/-}$ and wild-type mice to exhibit a comparable degree of paw swelling in response to capsaicin or carrageenan injection, suggesting that DREAM may not play an essential role in inflammation per se. Finally, in a model of neuropathic pain, chronic constriction of the sciatic nerve produced longlasting and robust mechanical allodynia in wild-type mice but not in dream $^{-/}$mice, which exhibited attenuated mechanical hypersensitivity to the peripheral neuropathy. Together, the data indicate that DREAM plays a pervasive as well as profound role in pain modulation in multiple tests of pain behaviour.

The hypoalgesic responses exhibited by dream $^{-/}$mice were attributed to enhanced $\kappa$-opioid tone. Both the pan-opioid antagonist, naloxone, and the $\kappa$-selective antagonist, norbinaltorphimine (nor-BNI), administered systemically were able to revert the pain behaviour of dream $^{-/-}$mice to that of wild-type controls in models of acute (noxious thermal and mechanical) and inflammatory (carrageenan) pain. Naloxone and nor-BNI partially rescued the hypoallodynic response of dream $^{-/-}$mice in the neuropathic model, when administered three weeks after initial nerve constriction. In addition, basal PDYN mRNA levels and dynorphin peptide content were higher in the spinal cords of $\mathrm{dream}^{-/-}$mice than in those of wild-type mice. ${ }^{25}$ These data suggest that increased basal activation of spinal $\kappa$-opioid receptors by their endogenous ligand, dynorphin peptides, is causal to the "ongoing analgesia" in mice lacking DREAM (see fig 1). However, non-opioid mechanisms may also contribute to the attenuated pain behaviour in dream $^{-/-}$mice in the neuropathic state, or, alternatively, ongoing activation of opioid receptors is not required for the hypoallodynia in these animals in the later stages of nerve injury. It is possible that the enhanced opioid tone at the initiation of nerve injury might reduce central sensitisation by either reducing the afferent drive via presynaptic mechanisms that inhibit excitatory neurotransmitter release, or by hyperpolarising second order dorsal horn neurones via a postsynaptic mechanism and reducing their excitability. Indeed, systemic administration of the NMDA receptor antagonist, MK-801, was found to partially reduce the tactile allodynia observed in wild-type neuropathic mice, but had no effect in dream $^{-/}$ neuropathic mice, indicating that NMDA receptor activation, or its behavioural consequences, is suppressed in the absence of DREAM.

\section{CONCLUSIONS}

Recent advances in pain research have begun to identify the mechanistic basis of chronic pain. In RA, it appears that peripheral nociceptors become sensitised due to an altered cytokine milieu or changes in the expression of ion channels, receptors, neurotransmitters, and neurotrophins. Central sensitisation is initiated in part by activation of key intracellular signal cascades bridging synaptic events to nuclear responses. Implicit in all of these events is the idea that any long term alteration in the nervous system involves molecular reprogramming of neurones through changes at the level of gene expression or transcription. A change in the expression of a particular gene may contribute directly to the pathophysiology or the development of the heightened pain state observed in patients. 
We have recently shown that DREAM is sufficient and necessary for transcriptional repression of the prodynorphin gene in spinal cord neurones. ${ }^{25}$ Lack of DREAM in mutant mice results in marked attenuation in pain behaviours regardless of the modality of the noxious stimuli or the tissue affected. Inactivation of DREAM also results in attenuation of inflammatory and neuropathic pain. Activation of the dynorphin selective $\kappa$-opiate receptors was found to be causal to the reduced pain responses in $\mathrm{dream}^{-/}$ mice. These findings provide a novel paradigm for the modulation of pain and identify DREAM as a critical transcriptional repressor for pain modulation. Importantly, lack of DREAM does not result in physical dependence on endogenous opioids. Thus, inhibition of DREAM might serve as a novel approach for the treatment of pain in the future.

\section{Authors' affiliations}

H-Y M Cheng, J M Penninger, IMBA, Institute for Molecular Biotechnology of the Austrian Academy of Sciences, Vienna, Austria H-Y M Cheng, J M Penninger, Departments of Medical Biophysics and Immunology and the University Health, Network, University of Toronto, Toronto, Ontario, Canada

Correspondence to: J Penninger, IMBA, Institute for Molecular Biotechnology of the Austrian Academy of Sciences, Dr Bohr Gasse 3-5, A-1030 Vienna, Austria; Josef.penninger@imba.oeaw.ac.at

\section{REFERENCES}

1 Feldmann M, Brennan FM, Maini RN. Role of cytokines in rheumatoid arthritis. Annu Rev Immunol 1996;14:397-440.

2 Woolf CJ, Salter MW. Neuronal plasticity: increasing the gain in pain. Science 2000;288:1765-9.

3 Gold MS, Levine JD, Correa AM. Modulation of TTX-R INa by PKC and PKA and their role in PGE2-induced sensitization of rat sensory neurons in vitro. J Neurosci 1998;18:10345-55.

4 Tominaga M, Caterina MJ, Malmberg AB, Rosen TA, Gilbert H, Skinner K, et al. The cloned capsaicin receptor integrates multiple pain-producing stimuli. Neuron 1998;21:531-43.

5 Shin J, Cho H, Hwang SW, Jung J, Shin CY, Lee SY, et al. Bradykinin-12lipoxygenase-VR1 signaling pathway for inflammatory hyperalgesia. Proc Natl Acad Sci U S A 2002;99:10150-5.

6 Maggi CA. The effects of tachykinins on inflammatory and immune cells. Regul Pept 1997;70:75-90.

7 von Banchet GS, Petrow PK, Braver R, Schaible HG. Monoarticular antigeninduced arthritis leads to pronounced bilateral upregulation of the expression of neurokinin 1 and bradykinin 2 receptors in dorsal root ganglion neurons of rats. Arthritis Res 2000;2:424-7.

8 Corsi MM, Ticozzi C, Netti C, Fulgenzi A, Tiengo M, Gaja G, et al. The effect of somatostatin on experimental inflammation in rats. Anesth Analg 1997:85:1112-15.
9 Helyes Z, Szabo A, Nemeth J, Jakab B, Pinter E, Banvolgyi A, et al. Antiinflammatory and analgesic effects of somatostatin released from capsaicin-sensitive sensory nerve terminals in a Freund's adjuvant-induced chronic arthritis model in the rat. Arthritis Rheum 2004;50:1677-85.

10 Morand EF, Leech M. Hypothalamic-pituitary-adrenal axis regulation of inflammation in rheumatoid arthritis. Immunol Cell Biol 2001;79:395-9.

11 Izeboud CA, Monshouwer M, van Miert AS, Witkamp RF. The betaadrenoceptor agonist clenbuterol is a potent inhibitor of the LPS-induced production of TNF-alpha and IL-6 in vitro and in vivo. Inflamm Res 1999;48:497-502.

12 Cronstein BN, Levin RI, Philips M, Hirschhorn R, Abramson SB, Weissmann G. Neutrophil adherence to endothelium is enhanced via adenosine $A 1$ receptors and inhibited via adenosine A2 receptors. J Immunol 1992;148:2201-6.

13 Wegener K, Kummer W. Sympathetic noradrenergic fibers as the source of immunoreactive alpha-neoendorphin and dynorphin in the guinea pig heart. Acta Anat (Basel) 1994;151:112-19.

14 Binder W, Mousa SA, Sitte N, Kaiser M, Stein C, Schafer M. Sympathetic activation triggers endogenous opioid release and analgesia within peripheral inflamed tissue. Eur J Neurosci 2004;20:92-100.

15 Miller LE, Justen HP, Scholmerich J, Straub RH. The loss of sympathetic nerve fibers in the synovial tissue of patients with rheumatoid arthritis is accompanied by increased norepinephrine release from synovial macrophages. FASEB J 2000; 14:2097-107.

16 Imai S, Tokunaga Y, Konttinen YT, Maeda T, Hukuda S, Santavirta S. Ultrastructure of the synovial sensory peptidergic fibers is distinctively altered in different phases of adjuvant induced arthritis in rats: ultramorphological characterization combined with morphometric and immunohistochemical study for substance $P$, calcitonin gene related peptide, and protein gene product 9.5. J Rheumatol 1997;24:2177-87.

17 Wu Z, Nagata K, lijima T. Immunohistochemical study of NGF and its receptors in the synovial membrane of the ankle joint of adjuvant-induced arthritic rats. Histochem Cell Biol 2000; 114:453-9.

18 Djouhri L, Dawbarn D, Robertson A, Newton R, Lawson SN. Time course and nerve growth factor dependence of inflammation-induced alterations in electrophysiological membrane properties in nociceptive primary afferent neurons. J Neurosci 2001;21:8722-33.

19 Neugebauer V, Lücke T, Schaible HG. N-methyl-d-aspartate (NMDA) and non-NMDA receptor antagonists block the hyperexcitability of dorsal horn neurones during development of acute arthritis in rat's knee joint. J Neurophysiol 1993;70:1365-77.

20 Carrion AM, Mellstrom B, Naranjo JR. Protein kinase A-dependent derepression of the human prodynorphin gene via differential binding to the intragenic silencer element. Mol Cell Biol 1998;12:6921-9.

21 Carrion AM, Link WA, Ledo F, Mellstrom B, Naranjo JR. DREAM is a $\mathrm{Ca}^{2+}$ regulated transcriptional repressor. Nature 1999;398:80-4.

22 Osawa M, Tong KI, Lilliehook C, Wasco W, Buxbaum JD, Cheng HY, et al. Calcium-regulated DNA binding and oligomerization of the neuronal calciumsensing protein, calsenilin/DREAM/KChIP3. J Biol Chem 2001;276:41005-13.

23 Buxbaum JD, Choi EK, Luo Y, Lilliehook C, Crowley AC, Merriam DE, et al Calsenilin: a calcium-binding protein that interacts with the presenilins and regulates the levels of a presenilin fragment. Nat Med 1998;4:1177-81.

24 An WF, Bowlby MR, Betty M, Cao J, Ling HP, Mendoza G, et al. Modulation of A-type potassium channels by a family of calcium sensors. Nature 2000;403:553-6.

25 Cheng HY, Pitcher GM, Laviolette SR, Whishaw IQ, Tong KI, Kockeritz LK, et al. DREAM is a critical transcriptional repressor for pain modulation. Cell 2002; 108:31-43. 